\title{
Manifestações oculares do transtorno do espectro alcoólico fetal: revisão bibliográfica Ocular manifestations of fetal alcohol spectrum disorders: a bibliographic review
}

\author{
Victor Ribeiro de Sant'Ana ${ }^{\circledR 1}$, Leonardo Marzola Hirata ${ }^{\circledR 1}$, Liane de Rosso Giuliani ${ }^{\circledR 1}$
}

\section{Resumo}

Introdução: O consumo de álcool por gestantes é reconhecidamente teratogênico, levando aos Transtornos do Espectro Alcoólico Fetal (TEAF). Indivíduos com esse espectro apresentam alterações em características faciais de nariz, lábios e orelhas, além das oculares. Objetivo: Realizar um levantamento de literatura científica para verificar as manifestações oculares presentes em pacientes diagnosticados com TEAF. Método: Foram pesquisados nos bancos de dados: Pubmed, Scielo, Cochrane e Lilacs artigos que trouxessem o tema descrito acima. Os dados obtidos da consulta foram tabulados com os seguintes parâmetros: estudo/ano, tipo de estudo/nível de evidencia, amostra e resultados. Resultados e Conclusão: De um total de 71 trabalhos, após análises de acordo com os critérios de inclusão e exclusão, foram selecionados 8. As principais manifestações oculares relacionadas à TEAF foram, em ordem decrescente: hipoplasia de nervo óptico, ambliopia, estrabismo, configuração anormal dos vasos retinianos, deficiência da função oculomotora, tamanho reduzido da órbita ocular, anormalidade na percepção de profundidade, displasia septo-óptica, astigmatismo e anisometropia. No tocante às manifestações oculares externas, as principais foram: blefarofimose, epicanto e telecanto.

Palavras chave: Transtornos do espectro alcoólico fetal, Oftalmopatias, Revisão

\section{Abstract}

Introduction: Alcohol consumption by pregnant women is knowned to be teratogenic, leading to Fetal Alcohol Spectrum Disorders (FASD). Individuals with this spectrum show changes in facial features of the nose, lips and ears, in addition to ocular ones. Objective: Perform a survey of scientific literature to verify the ocular manifestations present in patients diagnosed with FASD. Method: Pubmed, Scielo, Cochrane and Lilacs databases were searched

1. Universidade Federal de Mato Grosso do Sul. Faculdade de Medicina. Campo Grande-MS - Brasil

Trabalho realizado: Universidade Federal de Mato Grosso do Sul. Faculdade de Medicina. Campo Grande-MS - Brasil

Endereço para correspondência: Victor Ribeiro de Sant'Ana. Av. Sen. Antônio Mendes Canale, 1159, apto 201, bloco 8 - Pioneiros - 79070-295 - Campo Grande-MS - Brasil for articles that presented the theme described above. The data obtained from the consultation were tabulated with the following parameters: study / year, type of study/evidence level, sample and results. Results and Conclusion: From a total of 71 studies, after analysis according to the inclusion and exclusion criteria, 8 were selected. The main ocular manifestations related to FASD were, in decreasing order: optic nerve hypoplasia, amblyopia, strabismus, configuration abnormal retinal vessels, impaired oculomotor function, reduced size of the eye socket, abnormal depth perception, septo-optic dysplasia, astigmatism and anisometropia. With regard to external ocular manifestations, the main ones were: blepharophimosis, epicanthus and telecanto.

Keywords: Fetal alcohol spectrum disorders, Eye diseases, Review

\section{Introdução}

O consumo de bebidas alcoólicas por gestantes é reconhecido como um fator teratogênico que pode levar a alterações morfológicas e comportamentais variadas nos conceptos. A consequência mais grave desse consumo é designada como Síndrome Alcoólica Fetal (SAF). ${ }^{(1)}$ Contudo algumas crianças podem apresentar um fenótipo específico com a exposição ao etanol intra útero, mas sem se enquadrar necessariamente como SAF, sendo referidas pelo termo "Transtornos do Espectro Alcoólico Fetal (TEAF)", do inglês Fetal Alcohol Spectrum Disorders (FASD) ${ }^{(2-3)}$.

FASD abrange a síndrome alcoólica fetal (SAF), os defeitos congênitos relacionados ao álcool (Alcohol-Related Birth Defects - ARBD) e os distúrbios de neurodesenvolvimento relacionados ao álcool (Alcohol-Related Neurodevelopmental Disorders - ARND) ${ }^{(4)}$.

O álcool é capaz de atravessar a barreira placentária, contudo o embrião e a mãe não apresentam a mesma eficiência e abundância de enzimas para o metabolismo dessa substância. Isso permite que o álcool tenha um efeito prolongado sobre o feto levando a: diminuição dos níveis de antioxidantes endógenos, dano mitocondrial, peroxidação lipídica, interrupção da adesão das células neuronais, vasoconstrição placentária e inibição dos cofatores necessários para o desenvolvimento fetal ${ }^{(1)}$.

Vários indivíduos com TEAF apresentam características faciais anormais no nariz, lábios e orelhas ${ }^{(5)}$. 
Esses também podem ter o tamanho da cabeça, peso corporal e altura menor que a média além de alterações oculares ${ }^{(2,6)}$.

Mesmo a prevalência da TEAF sendo alta no mun$\mathrm{do}^{(7)}$, há poucos trabalhos que sintetizam as alterações oculares da doença.

\section{Objetivos}

O presente estudo se propõe a realizar um levantamento de literatura científica para verificar as manifestações oculares presentes em pacientes diagnosticados com desordens do espectro alcoólico fetal.

\section{Métodos}

Trata-se de uma revisão de literatura realizada após pesquisa nas bases de dados: Pubmed, Scielo, Cochrane e Lilacs.

Para a plataforma Pubmed, Scielo e Cochrane foram utilizados os descritores: "ocular"; "FASD"; e "Fetal Alcohol Spectrum Disorder", tendo sido encontrados 69 artigos. Na base de dados Lilacs, utilizou-se os descritores: "olho", "ocular", "transtornos do espectro alcoólico fetal" uma vez que não foram encontrados resultados na busca com os descritores em inglês supracitados, tendo sido obtidos 2 artigos.

Para a seleção dos artigos utilizados na revisão, foram utilizados os seguintes critérios de inclusão e exclusão.

Critérios de inclusão: estudos que abordam as repercussões oculares fetais devido a TEAF; estudos que abordam crianças já diagnosticadas com TEAF; artigos em português, inglês e espanhol; estudos publicados entre 2005 e 2020.

Critérios de exclusão: artigos baseados em estudos com animais; estudos que comparam as repercussões oculares em crianças expostas ao álcool durante a gestação mas que não foram diagnosticadas com TEAF; artigos que abordam somente as consequências maternas do uso de ácool durante a gestação; artigos que incluem outras drogas além do álcool; artigos que abordam exclusivamente intervenções para TEAF; artigos que já se compunham como revisão literária.

Os dados coletados foram tabulados para a análise descritiva.

\section{Resultados}

Após a primeira busca, foram obtidos um total de 71 artigos. Desses foram excluídos, após leitura do resumo, 57 artigos, por já não se enquadrarem aos critérios de inclusão e exclusão. Com um restante de 14, após leitura integral dos textos por 2 revisores, foram selecionados 8 artigos para serem utilizados no presente estudo.

Os dados obtidos através da análise dos 8 estudos selecionados foram agrupados em uma tabela (Quadro 1) de acordo com os seguintes parâmetros: estudo/ ano, tipo de estudo/ nível de evidência, amostra e resultados. Para a identificação do nível de evidencia de cada artigo foi utilizada a classificação de Oxford Centre for evidence-based medicine. ${ }^{(8)}$.

De acordo com os resultados mais descritos nos artigos, as principais manifestações oculares relacionadas à TEAF foram: hipoplasia de nervo óptico, ambliopia, estrabismo, configuração anormal dos vasos retinianos, deficiência da função oculomotora, tamanho reduzido da órbita ocular, anormalidade na percepção de profundidade, displasia septo-óptica, astigmatismo e anisometropia.

No tocante às manifestações oculares externas, as principais foram: blefarofimose; epicanto e telecanto.

\section{Discussão}

As manifestações morfológicas mais marcantes são encontradas geralmente quando o álcool é consumido pela mãe durante o primeiro trimestre de gravidez, a despeito de muitos efeitos teratogênicos que ainda ocorrerem nos dois últimos trimestres ${ }^{(9)}$.

Apesar da recomendação feita pela OMS de abstinência alcoólica durante a gestação devido a inexistência de uma margem segura definida na literatura, parecem existir 3 graus de exposição pré-natal ao etanol ingerido pela mãe. Esses 3 graus se distribuem em: grau elevado (48-60 g etanol/dia) - normalmente relacionado com a Síndrome Alcoólica Fetal; grau moderado (entre 24-48 g etanol/dia) - relacionando geralmente a outras desordens do espectro alcoólico fetal e exposições ocasionais ao álcool ${ }^{(10-11)}$.

Em Garcia et al ${ }^{(12)}$, há a proposição de que a prematuridade quando associada ao consumo de álcool pela gestante durante a gravidez constitui o risco associado mais comum para a hipoplasia de nervo óptico.

Foram encontradas também associações da TEAF com astigmatismo e anisometropia. Gummel, Ygge $^{(13)}$ indica grande tendência ao desenvolvimento de astigmatismo $(\mathrm{p}=0.0003)$, e boa possibilidade de anisometropia $(\mathrm{p}=0.027)$.

Paolozza et al ${ }^{(14)}$, trazem outra possível complicação do consumo de álcool durante a gestação: ocorre uma indução de lesão cerebral que resulta tanto em um baixo desempenho em medidas psicométricas como alterações no movimento ocular ${ }^{(14)}$. Entretanto, Tseng et $\mathrm{al}^{(15)}$, verificam que, apesar da deficiência da função oculomotora interferir no âmbito cognitivo, ela não é maior que o déficit de atenção que pode acompanhar a TEAF. 
Quadro 1

\begin{tabular}{|c|c|c|c|}
\hline \multicolumn{4}{|c|}{ Quadro 1} \\
\hline \multicolumn{4}{|c|}{ Dados obtidos através da análise dos 8 estudos selecionados. } \\
\hline Estudo/Ano & $\begin{array}{l}\text { Desenho de estudo } \\
\text { / Nivel de evidencia }\end{array}$ & Amostra & Resultados \\
\hline Vernescu et al (2012) ${ }^{(15)}$ & Caso controle / 4 & 21 casos / 21 controles & $\begin{array}{l}\text { Houve um padrão significativo de déficit de } \\
\text { visão semelhante a ambliopia nas crianças } \\
\text { com TEAF. }\end{array}$ \\
\hline Moore et al (2007) $)^{(18)}$ & Caso Controle / 4 & 118 casos / 158 controles & $\begin{array}{l}\text { O tamanho reduzido da órbita ocular foi } \\
\text { levanta do como um fator discriminante } \\
\text { para a TEAF. }\end{array}$ \\
\hline Gyllencreutz et al (2020) ${ }^{(2)}$ & Coorte / 3 & 30 pacientes & $\begin{array}{l}\text { Foi verificado que alterações oculares, como } \\
\text { estrabismo, anormalidades na percepção } \\
\text { da profundidade, configuração anormal de } \\
\text { vasos retinianos e hipoplasia de nervo óptico } \\
\text { são frequentemente presentes em pacientes } \\
\text { com TEAF e persistem até a idade adulta. }\end{array}$ \\
\hline Ribeiro et al (2007) ${ }^{(19)}$ & Caso Controle / 4 & 32 casos / 25 controles & $\begin{array}{l}\text { Foram encontradas principalmente anoma- } \\
\text { lias em seguimento posterior, destacando-se: } \\
\text { hipoplasia de do disco óptico e tortuosidade } \\
\text { dos vasos retinianos. }\end{array}$ \\
\hline Garcia et al (2006) $)^{(11)}$ & Coorte / 3 & 9 pacientes & $\begin{array}{l}\text { Todos os } 9 \text { pacientes possuíam hipoplasia } \\
\text { bilateral do nervo óptico. } 8 \text { possuíam estra- } \\
\text { bismo e } 2 \text { possuíam displasia septo-óptica. }\end{array}$ \\
\hline Paolozza et al(2013) $)^{(13)}$ & Caso controle / 4 & 72 casos / 139 controles & $\begin{array}{l}\text { Pacientes com TEAF apresentaram dificul- } \\
\text { dade de controlar os movimentos oculares } \\
\text { sacádicos, o que pode indicar regiões sobre- } \\
\text { postas do cérebro danificadas pela exposição } \\
\text { pré-natal ao álcool. }\end{array}$ \\
\hline Tseng et al $(2012)^{(14)}$ & Caso controle / 4 & 13 casos / 18 controles & $\begin{array}{l}\text { Crianças com TEAF possuem certa defi- } \\
\text { ciência na função oculomotora, mas menos } \\
\text { significativa que o déficit de atenção que a } \\
\text { síndrome carrega. }\end{array}$ \\
\hline Gummel, Ygge (2013) $)^{(12)}$ & Caso controle / 4 & 50 casos / 50 controles & $\begin{array}{l}\text { Quando analisadas quanto à acuidade visual, } \\
\text { as crianças com TEAF possuíam um maior } \\
\text { número de astigmatismo e anisometropia, } \\
\text { além de } 3 \text { casos de ambliopia. No tocante as } \\
\text { características oculares externas anormais, } \\
\text { foram achados } 17 \text { com blefarofimose, } 7 \text { com } \\
\text { epicanthus e } 16 \text { com telecanthus. Não foram } \\
\text { achadas ptoses palpebrais severas. Foram } \\
\text { encontrados discos ópticos inclinados em } 5 \\
\text { crianças com TEAF. Foi encontrado desali- } \\
\text { nhamento ocular em } 13 \text { crianças portadoras } \\
\text { de TEAF. }\end{array}$ \\
\hline
\end{tabular}

Em outro estudo, Vernescu et al ${ }^{(16)}$, avaliaram 21 crianças canadenses entre 6 e 11 anos diagnosticadas com TEAF. As crianças apresentaram déficit na visão espacial, incluindo acuidade visual, sensibilidade ao contraste e estereoacuidade.

Essa perda de padrão foi semelhante à ambliopia mas não superior as alterações ópticas e oculomotoras da experiência visual precoce que normalmente precedem essa condição como estrabismo e anisometropia $^{(16-17)}$.
Apesar dos sintomas acima descritos, não foram observadas anormalidades na visão de cores nesse estudo. Fato que é esperado em exposições a altos valores alcoólicos durante a gestação. Isso devido a redução da neurogênese, perda axonal, retinopatia diabética de início precoce, mielinização reduzida na retina e hipoplasia de nervo óptico ${ }^{(18)}$. Gyllencreutz et $\mathrm{al}^{(2)}$, em seu estudo prospectivo de coorte, verificaram que tais perdas neuronais podem persistir, inclusive, até a idade adulta. 
Sobre a distribuição das manifestações da TEAF de acordo com a etiologia, Moore et $\mathrm{al}^{(19)}$, em sua pesquisa, com um total de 118 casos, recrutados de 4 cidades distintas (Cidade do Cabo, Helsinque, Nova Iorque e San Diego) concluiram que cada etnia possui sua peculiaridade na apresentação da doença. Todavia a redução do tamanho da órbita ocular foi levantada como uma manifestação comum em todas as populações estudadas ${ }^{(19)}$.

Ribeiro et $\mathrm{al}^{(20)}$ revelam que os achados oculares mais comuns em pacientes com SAF foram estrabismo e anomalias do fundo da retina, particularmente hipoplasia do nervo óptico, assim como mostrado por Garcia et $\mathrm{al}^{(12)}$, e aumento da tortuosidade dos vasos retinianos, como já mostrado nos outros trabalhos citados $^{(20)}$.

Referente às manifestações oculares externas, o mesmo artigo evidenciou alguns achados que poderiam se relacionar a TEAF: fissura palpebral horizontal curta, epicanto, blefaroptose e telecanto ${ }^{(20)}$, sendo a fissura palpebral curta a única anormalidade ocular usada nos critérios diagnósticos para TEAF ${ }^{(21)}$.

Tais achados dialogam com o que foi mostrado por Gummel, Ygge ${ }^{(13)}$, em sua pesquisa realizada em um orfanato em São Petersburgo. Nesse trabalho, ela pôde notar a presença de blefarofimose cerca de quatro vezes maior que no grupo controle. Enquanto as manifestações de epicanto e telecanto foram encontradas em níveis até mesmo próximos de 8 vezes maiores que o do controle ${ }^{(13)}$.

\section{Conclusão}

A partir dessas relações, conclui-se que os etudos previamente citados sugerem que o consumo de álcool durante a gestação pode promover efeitos deletérios ao sistema visual. Sendo as principais manifestações por esses citadas: hipoplasia de nervo óptico, ambliopia, estrabismo, configuração anormal dos vasos retinianos e deficiência da função oculomotora. E quanto as manifestações oculares externas: blefarofimose; epicanto e telecanto. Toda essa sintomatologia oftálmica pode se enquandrar dentro da grande diversidade de sinais que a TEAF apresenta.

Vale ressaltar que o presente artigo verifica que a produção bibliográfica existente que correlacione de forma direta, e até mesmo indireta, as manifestações oculares com os transtornos do espectro alcoólico fetal é escassa.

Por fim, é clara a necessidade de um maior número de pesquisas e produções científicas nesse âmbito, com vistas a possibilitar a obtenção de correlações mais consistentes da sintomatologia ocular da TEAF e facilitar o seu diagnóstico.

\section{Referências}

1. Gupta KK, Gupta VK, Shirasaka T. An update on fetal alcohol syndrome pathogenesis, risks, and treatment. Alcohol Clin Exp Res. 2016; 40(8):1594-602.

2. Gyllencreutz E, Aring E, Landgren V, Svensson L, Landgren M, Grönlund M. Ophthalmologic findings in fetal alcohol spectrum disorders - a cohort study from childhood to adulthood. Am J Ophthalmol. 2020; 214:14-20.

3. Baptista FH, Rocha KBB, Martinelli JL, Avó LRS, Ferreira RA, Germano CMR, et al. Prevalence and factors associated with alcohol consumption during pregnancy. Rev Bras Saúde Mater Infant. [Internet]. 2017 [citado 2020 Mar 19]; 17(2): 271-9. Available from: http: / / www.scielo.br/scielo.php?script=sci_arttext\&pid=S1519-38292017000200271\&lng=en.

4. Sociedade de Pediatria de São Paulo. Grupo Álcool e Gravidez. Espectro de distúrbios fetais alcoólicos. Recomendações. Atualização de Condutas em Pediatria. [Internet]. 2015 [citado 2020 Maio 30]. (73):1-8. Disponível em:https://www.spsp.org.br/ site/asp/recomendacoes/Rec_73_Alcool.pdf

5. Wattendorf DJ, Muenke M. Fetal alcohol spectrum disorders. Am Fam Physician. 2005; 72(2):279-85.

6. Wilhoit LF, Scott DA, Simecka BA. Fetal alcohol spectrum disorders: characteristics, complications, and treatment. Community Ment Health. 2017; 53(6):711-8.

7. Lange S, Probst C, Gmel G, Rehm J, Burd L, Popova S. Global prevalence of fetal alcohol spectrum disorder among children and youth. JAMA Pediatr. 2017; 171(10):948-56.

8. OCEBM Levels of Evidence - CEBM [Internet]. CEBM. 2020 [acesso em 26 junho de 2020]. Disponível em: https://www. cebm.net/index.aspx?o=5653

9. Ornoy A. The impact of intrauterine exposure versus postnatal environment in neurodevelopmental toxicity: long-term neurobehavioral studies in children at risk for developmental disorders. Toxicol Lett. 2003; 140-141:171-81.

10. Martínez-Frías M, Bermejo E, Rodríguez-Pinilla E, Frías J. Risk for congenital anomalies associated with different sporadic and daily doses of alcohol consumption during pregnancy: a case-control study. Birth Defects Res A Clin Mol Teratol. 2004; 70(4):194-200

11. Dawson D, Das A, Faden VB, Bhaskar B, Krulewitch CJ, Wesley B. Screening for high- and moderate-risk drinking during pregnancy: a comparison of several TWEAK-based screeners. Alcohol Clin Exp Res. 2001; 25(9):1342-9.

12. Garcia ML, Ty EB, Taban M, Rothner A, Rogers D, Traboulsi EI. Systemic and ocular findings in 100 patients with optic nerve hypoplasia. J Child Neurol. 2006; 21(11):949-56.

13. Gummel K, Ygge J. Ophthalmologic findings in Russian children with fetal alcohol syndrome. Eur J Ophthalmol. 2013; 23(6):82330.

14. Paolozza A, Rasmussen C, Pei J, Hanlon-Dearman A, Nikkel SM, Andrew G, et al. Deficits in response inhibition correlate with oculomotor control in children with fetal alcohol spectrum disorder and prenatal alcohol exposure. Behav Brain Res. 2014; 259:97-105.

15. Tseng PH, Cameron IG, Pari G, Reynolds JN, Munoz DP, Itti L. High-throughput classification of clinical populations from natural viewing eye movements. J Neurol. 2012; 260(1):275-84.

16. Vernescu RM, Adams RJ, Courage ML. Children with fetal alcohol spectrum disorder show an amblyopia-like pattern of vision deficit. Dev Med Child Neurol. 2012; 54(6):557-62.

17. DeSantis D. Amblyopia. Pediatr Clin North Am. 2014; 61(3):50518 .

18. Stromland K, Pinazo-Durán MD. Ophthalmic involvement in the fetal alcohol syndrome: clinical and animal model studies. Alcohol Alcohol. 2002; 37(1):2-8. 
19. Moore ES, Ward RE, Wetherill LF, Rogers JL, Autti-Rämö I, Fagerlund $\AA$, et al. Unique facial features distinguish fetal alcohol syndrome patients and controls in diverse ethnic populations. Alcohol Clin Exp Res. 2007; 31(10):1707-13.

20. Ribeiro IM, Vale PJ, Tenedório PA, Rodrigues PA, Bilhoto MA, Pereira HC. Ocular manifestations in fetal alcohol syndrome. Eur J Ophthalmol. 2007; 17(1):104-9.
21. Hoyme HE, Kalberg WO, Elliott AJ, Blankenship J, Buckley D, Marais AS, et al. Updated clinical guidelines for diagnosing fetal alcohol spectrum disorders. Pediatrics. 2016; 138(2):e20154256.

Trabalho recebido: 31/05/2019

Trabalho aprovado: 01/07/2020

Trabalho publicado: 01/07/2020 